\title{
Treating The Triad: Teeth, Muscles, TMJs
}

\section{Giuseppe Cozzani}

Quintessence Publishing Company, Inc

Language: English

408 pages, 1707 illustrations

ISBN: 978-88-7492-152-2

Publication Date: March, 2011

Price: $248.00 €$

If we carefully follow the specialty literature we'll find that over time many books which addressed the function and dysfunction of TMJ were published. The interest raised by this book written by Dr. Giuseppe Cozzani, specialist in orthodontics, is to discuss the relationship between teeth, muscles and TMJ focusing on the diagnosis, on the principles of maintaining or recovery of the stomatognathic system function as part of orthodontic treatment, particularly in dealing with facial pain and postural problems. The author achieves a systematic approach of this group of disorders planning the treatment, from the simpler to the most complex cases, in two phases: Musculoarticular Therapy and Orthodontic Occlusal Finishing.

The book contains 408 pages and is divided into six distinct chapters.

The first chapter, "Basic Concepts", introduces us in the anatomy and pathophysiology of the stomatognathic system for a better understanding of the philosophy of the Temporomandibular Disorders (TMDs) treatment, presenting us the temporomandibular joints, the true articulating surface, the skeletal muscle apparatus, the basic muscle anatomy, the axial alignment of joint structures, the old and new concept of centric relation, the physiology of jaw opening and closure, the anatomical parts involved in mastication, the tooth dynamics and esthetics .

In the second chapter, "Diagnosis: Patient Records", the patient assessment, the basic medical history, clinical records, clinical analysis, medical imaging, imaging diagnostics of TMJs, the importance of checking 3D mandibular movements, TMJ palpation and auscultation and diagnostic - therapeutic manual techniques are presented.

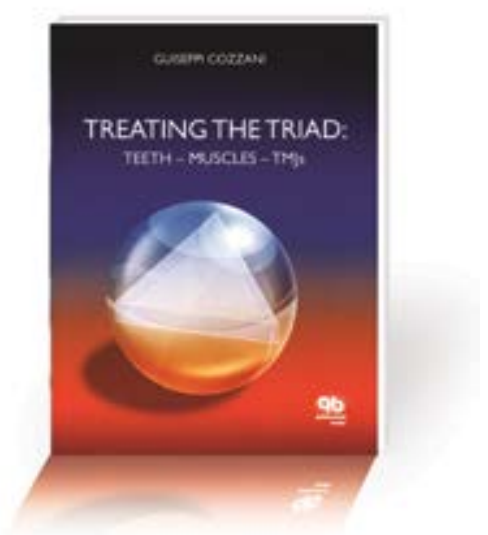

After the completion of all the examinations and tests, the clinician should be able to reach a clear diagnosis and plan in the next chapter, "Phase I: Musculoarticular Therapy" that describes considerations on compromise and adaptability, coordination between articular eminence inclination, cusp inclination and incisal guidance, assessment of changes in pretreatment and posttreatment condylar position, bite registration, splints, therapeutic procedure sequence, pain, TMD and postural disorders, occlusion, posture and MRI, musculodental extracapsular pathology, intermediate pathology, intracapsular pathology: dislocation with and without reduction, intracapsular pathology: destruction, PHASE I: virtual ARS musculoarticular rehabilitation treatment and intracapsular pathology: condylar hypermobility and ligamentous laxity

The author also discusses Phase II: Orthodontic Occlusal Finishing, its principal aim being to obtain a correct interarch relationship in harmony with the muscle and joints considering other joints and the TMJ, temporomandibular disorders in children including case presentations as well as the innovative orthodontic treatment of teeth, muscles and temporomandibular disorders.

For a better understanding, the text comes with 1707 illustrations, diagrams, MRI and clinical photographs, constituting an essential reference for the orthodontists interested in the treatment of temporomandibular disorders (TMDs). 